\section{Relationship between cerebrovascular reactivity and age in multiple sclerosis}

\author{
Laura Pelizzari, ${ }^{1}$ \\ Maria Marcella Laganà, ${ }^{1}$ \\ Laura Mendozzi, \\ Niels Bergsland, ${ }^{1,2}$ Pietro Cecconi, ${ }^{1}$ \\ Stefano Viotti, ${ }^{1}$ Luigi Pugnetti, ${ }^{1}$ \\ Raffaello Nemni, ${ }^{1,3}$ Giuseppe Baselli, ${ }^{4}$ \\ Mario Clerici, ${ }^{1,3}$ Francesca Baglio ${ }^{1}$ \\ ${ }^{1}$ IRCCS, Fondazione Don Carlo \\ Gnocchi, Milano, Italy; ${ }^{2}$ Buffalo \\ Neuroimaging Analysis Center, \\ Department of Neurology, Jacobs School \\ of Medicine and Biomedical Sciences, \\ University at Buffalo, State University of \\ New York, Buffalo, NY, USA; \\ ${ }^{3}$ Department of Physiopathology and \\ Transplants, University of Milano, \\ Milano, Italy; ${ }^{4}$ Department of \\ Electronics, Information and \\ Bioengineering, Politecnico di Milano, \\ Milano, Italy
}

\section{Background}

Cerebrovascular reactivity (CVR) alterations have been observed within gray matter (GM) in multiple sclerosis (MS) patients. ${ }^{1,2}$ This study aimed to: i) test alterations of cerebral blood flow (CBF) and CVR in MS, within GM and white matter (WM) regions of interest (ROIs); ii) assess CVR within MS WM lesions; iii) investigate the association between CVR and age in MS.

\section{Methods}

Thirty-one MS patients (median age $[\mathrm{IQR}]=39.8$ [33.3-51.3] years old, median EDSS $[\mathrm{IQR}]=1.5[1.0-3.0])$ and 25 healthy controls (HC) (median age $[\mathrm{IQR}]=42.3$ [29.3-56.7] years old) were scanned with pseudo-continuous arterial spin labeling MRI using a $1.5 \mathrm{~T}$ scanner, once under normocapnia and once under hypercapnia. CBF images were computed, ${ }^{3}$ and partial volume effect correction was performed. CVR maps were derived. ${ }^{1}$ Median CBF (at normocapnia) and CVR were extracted from GM atlas-derived functional ROIs, ${ }^{2}$ GM vascular territory ROIs, WM lesions and normal appearing WM (NAWM). MS$v s-\mathrm{HC} \mathrm{CBF}$ and CVR differences were assessed in all the ROIs. Spearman's correlations between CVR and age were computed for MS and $\mathrm{HC}$ groups. The false discovery rate (FDR) method was used to correct for multiple comparisons.

\section{Results}

No significant MS- $v s$-HC differences were observed for $\mathrm{CBF}$ and CVR. Significantly lower CBF, but not CVR, was found in WM lesions with respect to NAWM $(\mathrm{P}<0.0001)$ in MS group. A significant FDR-corrected inverse correlation between CVR and age was observed in the HC group for all GM ROIs and WM (e.g. within somatomotor network, $r=-0.437$, $\mathrm{P}=0.031 ; \mathrm{WM}, \mathrm{r}=-0.488, \mathrm{P}=0.025)$, but not in MS group.

\section{Conclusions}

The relatively low-grade of inflammation in our MS patients may have prevented us from showing significant CVR differ-
Correspondence: Laura Pelizzari, IRCCS, Fondazione Don Carlo Gnocchi, Milano, Italy. E-mail: lapelizzari@dongnocchi.it

Key words: Neurovascular diseases; meeting.

Conference presentation: $9^{\text {th }}$ Annual Meeting of the International Society of Neurovascular Disease (ISNVD), May 30 $30^{\text {th }}-31^{\text {st }}, 2019$, Ferrara, Italy.

This work is licensed under a Creative Commons Attribution 4.0 License (by-nc 4.0).

CCopyright: the Author(s), 2019

Licensee PAGEPress, Italy

Veins and Lymphatics 2019; 8:8429

doi:10.4081/vl.2019.8429

ences between MS and HC groups. Since CVR and age were correlated in HC group but not in the MS one, some other factors, that may be MS-related, could be associated with CVR in MS patients.

\section{References}

1. Marshall $\mathrm{O}, \mathrm{Lu} \mathrm{H}$, Brisset $\mathrm{JC}$, et al. Impaired cerebrovascular reactivity in multiple sclerosis. JAMA Neurol 2014;71:1275-81.

2. Marshall O, Chawla S, Lu H, et al. Cerebral blood flow modulation insufficiency in brain networks in multiple sclerosis: A hypercapnia MRI study. J Cereb Blood Flow Metab 2016;36: 2087-95.

3. Laganà MM, Mendozzi L, Pelizzari L, et al. Are cerebral perfusion and atrophy linked in multiple sclerosis? Evidence for a multifactorial approach to assess neurodegeneration. Curr Neurovasc Res 2018;15:282-91. 